\title{
Concept for accelerated ray-based monostatic RCS simulations using bistatic approximations
}

\author{
H. Buddendick ${ }^{1}$ and T. F. Eibert ${ }^{2}$ \\ ${ }^{1}$ Institute of Radio Frequency Technology, Universität Stuttgart, 70550 Stuttgart, Germany \\ ${ }^{2}$ Lehrstuhl für Hochfrequenztechnik, Technische Universität München, 80290 München, Germany
}

\begin{abstract}
An approach enabling accelerated shooting and bouncing rays (SBR) simulations to determine the backscattering properties of electrically large and complex objects is presented. Instead of performing independent simulations for all required aspect angles, the concept is based on the idea of additionally exploiting bistatic information for some neighboring aspect angles. Therefore the results of the geometrical ray tracing, which consumes a large part of the computational resources in case of complex shaped objects can be reused multiple times with only low additional computational resources.

This method works well for objects with a sufficiently smooth shape and if a large number of aspect angles is to be simulated. A simple generic simulation example is used to show the general applicability of the method and to examine the degradation of the results depending on the applied bistatic angle. Furthermore, the acceleration that can be expected by the presented approach is determined and verified with the simulation example.
\end{abstract}

\section{Introduction}

The Radar Cross Section (RCS) is an important characterization for the electromagnetic scattering properties of complex objects. It is common practice to use this quantity in the fields of radar system design and dimensioning, as well as for radar algorithm development and validation. In many areas it is desired to have a good estimation for it for a wide range of aspect angles. Unfortunately, it is computationally very demanding to perform the required simulations for complex shaped objects like vehicles or airplanes at very high frequencies, even if suitable high frequency approximations like the

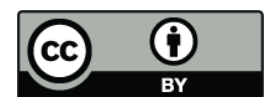

Correspondence to: H. Buddendick (buddendick@ihf.uni-stuttgart.de) widely used Geometrical Optics (GO) and Physical Optics (PO) are used.

Based on a well known relationship for the scattering properties at different aspect angles (sometimes referred to as bistatic monostatic equivalence principle) we present an approach which enables us to accelerate considerably these raybased multi-aspect RCS simulations.

Basically, in SBR rays representing the incident fields are used to determine the resulting surface currents and the resulting field contributions at the given observation points according to the Physical Optics approach. This is explained in Sect. 2 with special focus on those aspects that are interesting for the application of the bistatic monostatic equivalence principle which is introduced in the first part of the following Sect. 3. The second part of Sect. 3 demonstrates how, with a proper phase correction, contributions related to deviating illumination and observation angles can be utilized for an approximation of the desired monostatic RCS. In this way from each determined ray, contributions to several neighboring monostatic receivers can be obtained, and therefore the total number of rays required for a proper sampling of the object can be reduced significantly.

In Sect. 4 some remarks about the angular sampling are given, as it has a very important influence on the efficiency of the presented method. In Sect. 5 simulation results are presented to demonstrate the applicability and performance of the approach and, finally, Sect. 6 summarizes the results we have obtained up to now.

\section{The applied shooting and bouncing rays technique}

To analyze the scattering properties of electrically large and complex objects asymptotic high frequency methods are to be applied. In this paper we use an approach which is based on a shooting and bouncing rays (SBR) algorithm to determine the local incident fields on the scatterer and the Physical 


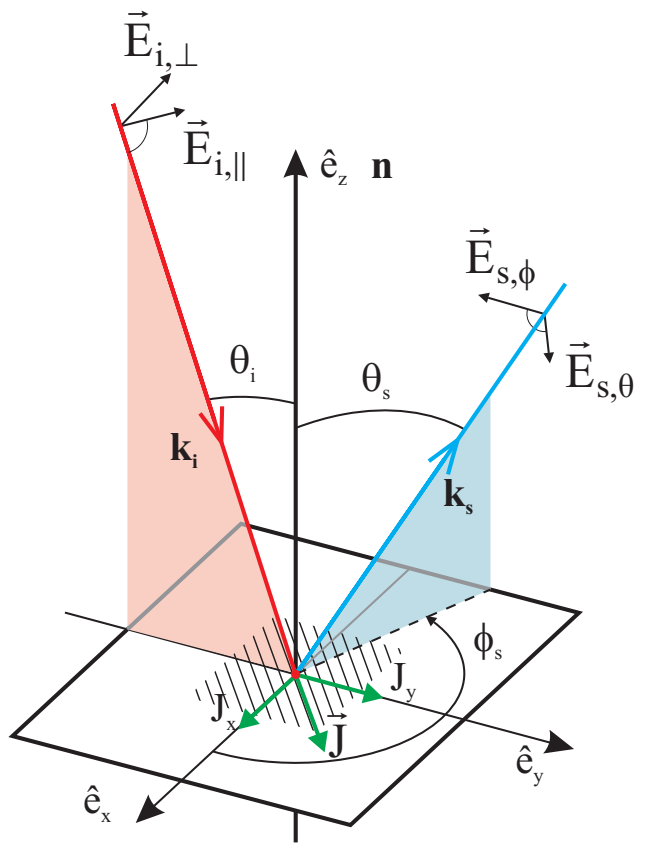

Fig. 1. Local coordinate system used for the PO integration around the interaction point of a ray on a plane surface element.

Optics (PO) to determine the resulting field contributions. In the SBR approach, see for example Ling et al. (1989), a number of rays is launched towards the scatterer and the rays are traced with one or more interactions until they exit the scenario, so that the fields at the last interaction point can be used to determine the surface currents. Nowadays, the concept of SBR is widely applied to compute the scattering fields of large objects in scientific as well as in commercial codes. Some examples can be found in Weinmann (2006); Galloway and Welsh (2005); Andersh et al. (2000).

Basically, our algorithm splits into two parts:

- Ray tracing is used to find the fields on the objects surface according to the GO principle.

- PO integration determines the scattered fields based on the surface currents.

An important observation is that usually the ray tracing part of the algorithm consumes most of the computational resources if detailed object models are considered. For typical applications the complexity of the geometric model can be in the order of a few 100000 triangles. This clearly indicates the need for a very efficient implementation, which, in our case, is based on a uniform spatial subdivision (Fujimoto, 1986).

The number of rays that are directed towards the object must be large enough to capture all relevant object details sufficiently for the coherent superposition of the field contributions. In our case a small random component is added to

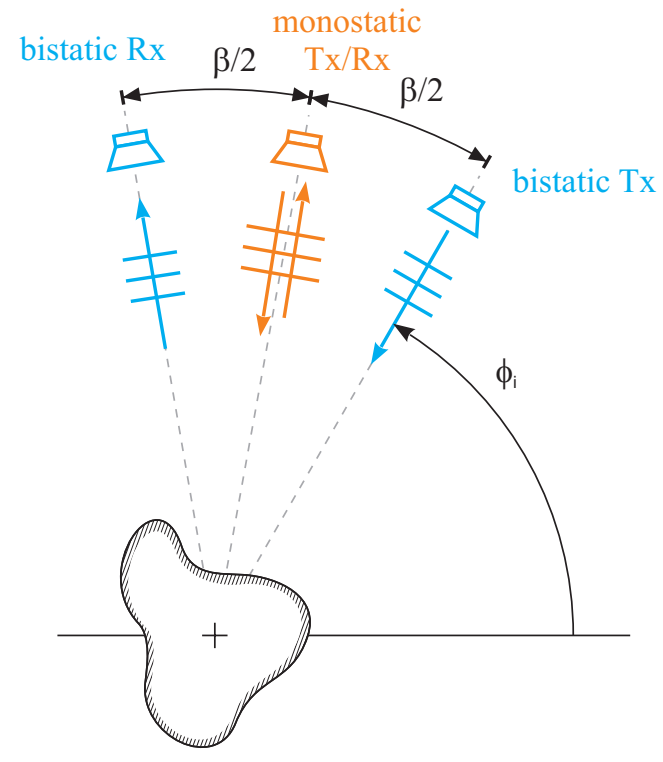

Fig. 2. Equivalence of bistatic and monostatic RCS.

the ray directions to prevent aliasing effects at surface borders or wedges. Due to this stochastic ray launching process the results of two independent simulation runs (with identical parameter settings) will differ from each other and include a stochastic component as well. With an increasing number of rays this deviation will decrease and the simulations will converge. Obviously, the stochastic uncertainty will be largest for low scattering levels and the expected accuracy can be used to adjust the required number of rays.

The scattering contributions are determined from the last interaction point of the ray on the object. According to Fig. 1 a local coordinate system adapted to the current ray is used and the surface currents can be computed in the following form:

$J_{y}=2 \hat{n} \times \boldsymbol{H}_{i, \|}=\frac{2}{Z_{F 0}} E_{i, \|} \hat{e}_{y}$,

$J_{x}=2 \hat{n} \times \boldsymbol{H}_{i, \perp}=\frac{-2 \cos \theta_{i}}{Z_{F 0}} E_{i, \perp} \hat{e}_{x}$.

Finally, the far-field scattering contributions caused by these currents can be derived from the wave equation in two orthogonal polarization directions at the given observation points. For details the reader may refer to Balanis (1989), from where we have adopted the notation.

\section{Description of the acceleration approach}

\subsection{Monostatic bistatic equivalence}

Considering the scattering properties of an object and a geometrical setup as shown in Fig. 2, a simple and well known 


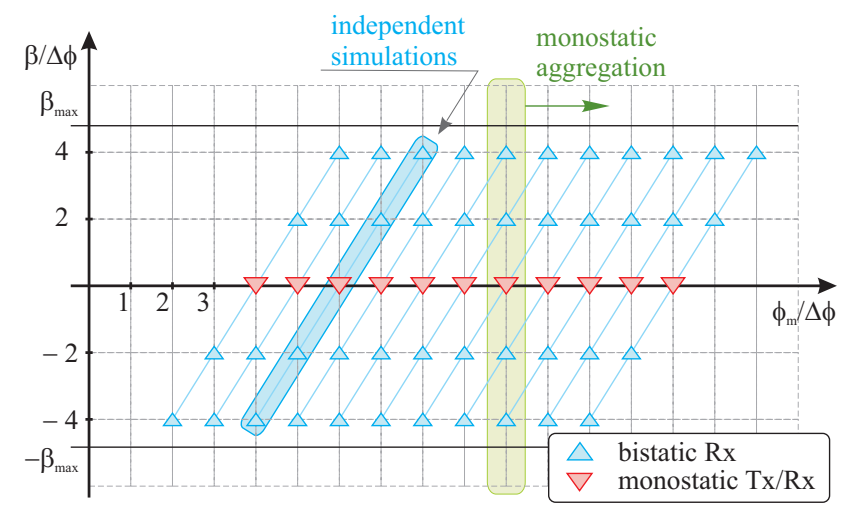

Fig. 3. Symmetric distribution of temporary bistatic receivers around the monostatic receivers and final monostatic aggregation.

relationship between bistatic and monostatic RCS values exists and can be expressed in the following form:

$\sigma_{m}\left(\phi_{i}+\beta / 2\right)=\sigma_{b}\left(\phi_{i}, \beta\right)$,

with $\sigma_{m}$ and $\sigma_{b}$ being the monostatic and bistatic RCS, respectively, and $\phi_{i}$ the angle of incidence and $\beta$ the bistatic angle. This means that the bistatic RCS equals the monostatic RCS observed at the bisecting angle (see Fig. 2). The underlying assumptions for this identity are the consideration of very high frequencies and a sufficiently smooth surface of the scattering object. Details on the monostatic bistatic equivalence including an analytical discussion can be found in Kell (1965) and Siegel et al. (1968). Several interesting applications of the aforementioned principle can be found. Some applications are in the context of RCS measurements, e.g. monostatic RCS values derived from bistatic measurement setups, or, in the reverse direction, bistatic RCS derived from monostatic measurements (Kell, 1965). In the field of simulation techniques the equivalence has been successfully used to accelerate FVTD, FDTD, or MoM codes in a postprocessing manner, see for example Schuh et al. (1994). According to the authors knowledge no publication on the use of the bistatic monostatic equivalence on a direct ray-by-ray basis exists.

Another interesting use of this principle is related to the possibility of generating multi-frequency data from multiaspect (bistatic) data. This can be advantageously utilized in the field of Synthetic Aperture Radar (SAR) imaging as described for example by Mensa (1991). As these methods are based on a coherent signal processing, a simple power based formulation as given in Eq. (3) is not sufficient, and a coherent scattering model has to be used instead. Similarly, in the presented SBR approach rays are added up coherently and consequently a coherent scatterer model is used to analyze the monostatic and bistatic relation. This results in a simple but important phase correction factor to be considered for bistatic contribution as derived in the following section.

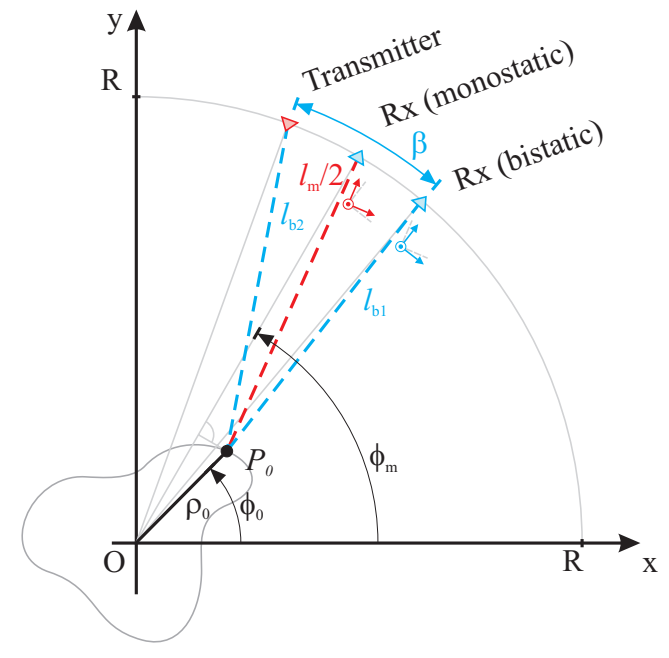

Fig. 4. The actual bistatic path differs from the monostatic path for which the contribution is to be considered and the phase has to be corrected accordingly.

\subsection{Bistatic acceleration of the SBR algorithm}

Figure 3 shows the basic transmitter (Tx) and receiver ( $\mathrm{Rx})$ arrangement that is used to capture the bistatic information during the SBR algorithm. For each desired observation direction a conventional monostatic $\mathrm{Tx} / \mathrm{Rx}$ pair is placed equally spaced by $\Delta \phi$ in the angular domain. Additionally, for each simulation a number of temporary bistatic receivers is created symmetrically around the desired observation points, each separated by $2 \Delta \phi$. The bistatic reuse factor $N_{b i}$ equals the number of monostatic observation angles within a sector $\beta_{\max }$ :

$N_{b i}=2\left\lfloor\frac{\beta_{\max }}{2 \Delta \phi}\right\rfloor+1$.

After all observation directions have been simulated, the contributions captured by the bistatic receiver can be converted to the bisecting monostatic receiver (this is called monostatic aggregation here). In this way the rays launched from each monostatic direction are reused for several neighboring directions as well. Consequently, for the same accuracy as discussed in Sect. 2, the number of rays launched for each observation direction can be reduced significantly.

During the SBR algorithm a bistatic field contribution is determined for all rays at the location of all temporary bistatic receiver within the bistatic sector defined by $\beta_{\max }$ symmetrically around the transmitter.

Now let's assume for a coherent consideration a point scatterer on the surface of the object as depicted in Fig. 4. The length of the actual bistatic path $l_{b}=l_{b 1}+l_{b 2}$ of the ray differs from the length $l_{m}$ of the path to the bisecting monostatic observation point to which the ray finally shall contribute according to the bistatic equivalence principle. Due to this path 
Table 1. Examples for the required angular sampling interval.

\begin{tabular}{c|c|ccc}
\hline $\begin{array}{c}f \\
{[\mathrm{GHz}]}\end{array}$ & $\begin{array}{c}\rho_{\max } \\
{[\mathrm{m}]}\end{array}$ & $\begin{array}{c}\Delta \phi_{\max } \\
{\left[{ }^{\circ}\right]}\end{array}$ & $\begin{array}{c}N_{\phi} \\
\phi=0 . .180^{\circ}\end{array}$ & $\begin{array}{c}N_{b i} \\
\beta_{\max }=10^{\circ}\end{array}$ \\
\hline 10 & 0.09 & 4.77 & 38 & 3 \\
10 & 0.9 & 0.477 & 377 & 21 \\
24 & 2.5 & 0.0716 & 2514 & 139 \\
80 & 2.5 & 0.0215 & 8378 & 465
\end{tabular}

length difference a phase correction is required. For a far field observation distance with $R \gg \rho_{0}$ the path length difference depending on the coordinates of $P_{0}$ and the observation point can be determined to be

$\Delta l=l_{m}-l_{b}=-2 \rho_{0} \cos \left(\phi_{m}-\phi_{0}\right)(1-\cos (\beta / 2))$,

or, alternatively depending only on the quantities directly accessible in the simulation:

$\Delta l=\left(l_{b}-2 R\right)\left(\frac{1}{\cos (\beta / 2)}-1\right)$.

Following this model a simple phase correction corresponding to

$\Delta \psi=k_{0} \Delta l$,

with the free-space wave number $k_{0}=2 \pi / \lambda_{0}$ is applied to all field contributions on a ray-by-ray basis. Furthermore it can be seen from Fig. 4 that the coordinate system of the (temporary) bistatic receiver is rotated by $\beta / 2$. This should not be a problem as it must be done only once at the end, when the coherent sum of the temporary bistatic receiver is finally added to the corresponding monostatic receiver during the monostatic aggregation.

\subsection{Expected performance and limitations}

Obviously, the use of the bistatic monostatic equivalence principle limits the presented approach to high frequency applications and to sufficiently smooth objects. The first restriction is not very stringent as this is the main focus of this acceleration approach anyway, and it scales well with its necessity and efficiency, as it will be shown later. The phase correction as explained above degrades in case of multiple bounces (double reflection). This is also closely related to the smoothness of the object and therefore not a new restriction. Here, the smoothness of the object is partly related to a similar illumination of the object for the bistatic and the monostatic case. This may be effected by exposed geometrical features causing a self-shadowing on the object itself. The example in Sect. 5 shows how the smoothness of the object effects the results.

The acceleration that can be expected could be in the range of the bistatic reuse factor $N_{b i}$ as this is the factor by which

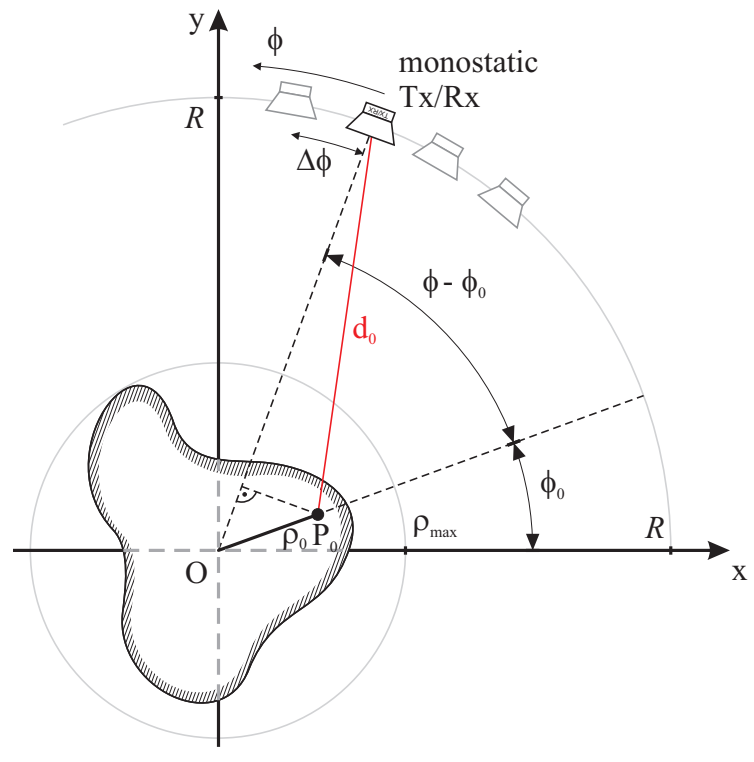

Fig. 5. Geometry for the determination of the minimum angular sampling rate. A point source $P_{0}$ on the objects surface is assumed.

the number of effectively evaluated rays exceeds the number of distinct rays. Consequently, the number of rays required to achieve results comparable with pure monostatic simulations could be lowered by $N_{b i}$ and for realistic scenarios the required CPU time is proportional to the number of rays.

Unfortunately, the improvement that can be realized will be somewhat lower. On the one hand this is due to a degradation in the quality of the contribution of the rays with larger bistatic angles. This will lead to systematic error contributions. On the other hand an additional overhead in both, memory and computational cost, due the temporary bistatic receiver will decrease the theoretical performance. Hence, the resulting acceleration that can be achieved will be below the bistatic reuse factor $N_{b i}$. Furthermore, as each observation point contains (bistatic) information originating from neighboring transmitters, the simulated observation range has to be extended by $\beta_{\max } / 2$ at both ends to capture the bistatic information symmetrically. But for many practical scenarios with large observation ranges this has only minor impact.

\section{Angular sampling}

From the preceding sections we can conclude that the efficiency that can be expected from the bistatic acceleration depends on the bistatic reuse factor $N_{b i}$ and as the maximum bistatic angle $\beta_{\max }$ is determined only by the geometry of the object and is therefore fixed, the resulting efficiency depends on the applied (monostatic) sampling rate. Often it is desired to have a full representation of the backscattering fields 


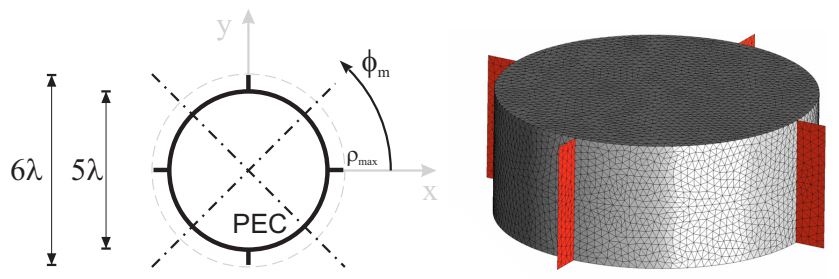

Fig. 6. Generic object representing a smooth body (cylinder) with blades causing bistatic shadowing and double reflections.

of an object for a certain aspect range, e.g. in the azimuthal plane. In this case the required observation angles are to be determined according to the rules of the sampling theorem. Assuming far field conditions an angular sampling interval of

$\Delta \phi_{\max }=\frac{\lambda_{0}}{4 \rho_{\max }}$

is required, with $\lambda_{0}$ being the carrier wavelength and $\rho_{\max }$ the maximum object radius in the considered observation plane. Some sampling intervals and the resulting $N_{b i}$ for an bistatic sector of $\beta_{\max }=10^{\circ}$ are given in Table 1 . Note that at frequencies as high as $80 \mathrm{GHz}$ objects of the size of a passenger car require a large amount of observation directions according to the sampling theorem. On the other hand the increasing bistatic reuse factor $N_{b i}$ offers a compensation for this enormous computational burden of such problems at very high frequencies. For the derivation of Eq. (8) we assume a scattering model with a geometrical setup as shown in Fig. 5 with a point scatterer $P_{0}$ on the surface of an object. For large observation distances $R$ the amplitude of the field contributions at the observation point is assumed to be constant and the phase is proportional to the path length

$\psi\left(d_{0}\right)=k_{0} 2 d_{0}(R, \phi)$

with $d_{0}$ being the distance between $P_{0}$ and the Tx/Rx location. Similar to time dependent signals, a variability $f_{\phi}$ in the $\phi$-domain can be obtained from the derivative of the phase according to the $\phi$ angle:

$2 \pi f_{\phi}=\frac{\partial}{\partial \phi} \psi\left(d_{0}\right)=2 k_{0} \frac{\partial}{\partial \phi} d_{0}$.

Here, the geometrical path length is given by

$d_{0}(R, \phi)=\sqrt{R^{2}+\rho_{0}^{2}-2 \rho_{0} R \cos \left(\phi-\phi_{0}\right)}$,

$\frac{\partial}{\partial \phi} d_{0}(R, \phi)=\frac{-\rho_{0} R \sin \left(\phi-\phi_{0}\right)}{\sqrt{R^{2}+\rho_{0}^{2}-2 \rho_{0} R \cos \left(\phi-\phi_{0}\right)}}$.

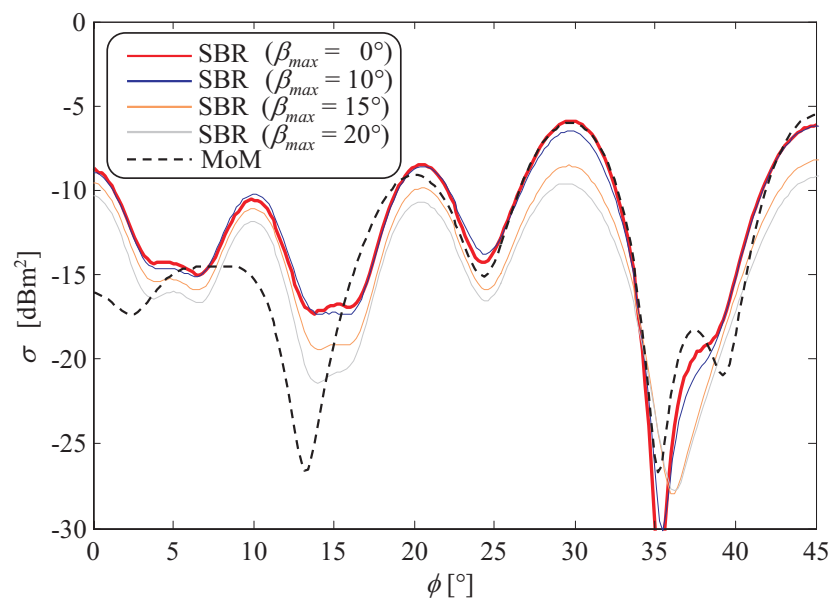

Fig. 7. RCS of the generic cylinder model with different levels of bistatic information.

According to the Nyquist sampling theorem a complete signal representation requires observation angles with a resolution below

$\Delta \phi_{\max }=\frac{1}{2 f_{\phi, \max }}=\frac{\lambda_{0}}{4 \max \left[\left|\frac{\partial}{\partial \phi} d_{0}\right|\right]}$.

Obviously, the maximum in Eq. (13) is achieved for $\left|\phi-\phi_{0}\right|=\pi / 2$, i.e. for $P_{0}$ having the largest lateral distance to the central observation line:

$\max _{\phi}\left[\left|\frac{\partial}{\partial \phi} d_{0}(R, \phi)\right|\right]=\frac{R \rho_{\max }}{\sqrt{R^{2}+\rho_{\max }^{2}}}$

This leads to

$\Delta \phi_{\max }=\frac{\lambda_{0} \sqrt{R^{2}+\rho_{\max }^{2}}}{4 R \rho_{\max }}$,

which, for the far field with $R \rightarrow \infty$, directly results in Eq. (8).

\section{Simulation results for a generic test object}

To determine the effect of the bistatic approximation in case of a non-smooth object including self-shadowing features, a small metal cylinder with four radial blades is considered as depicted in Fig. 6. With the given relative dimensions of the blades, the resulting shadows on the object will be much more penalizing for our approach than what is expected from a realistic object like a passenger car. Besides these shadowing effects multiple reflections will occur between the blades and the cylinder. Therefore, we conclude that this object, qualifies well for a test case and to derive an upper bound for $\beta_{\text {max }}$.

Due to symmetry only a $45^{\circ}$ sector must be considered for the monostatic RCS. Figure 7 shows in red the RCS obtained with the straightforward (not accelerated) SBR for a 


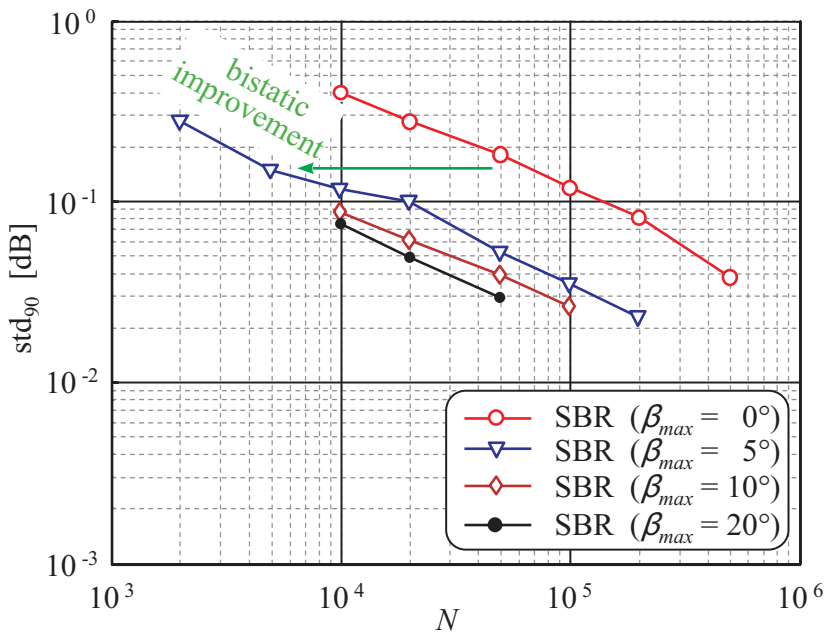

Fig. 8. Influence of the bistatic information on SBR convergence. Standard deviations vs. number of rays for different $\beta_{\max }$.

very high number of rays. This can be considered as reference of what can be achieved with the SBR approach. Additionally, the results from a Method of Moments (MoM) implementation is shown as dashed line. A good agreement for the basic scattering characteristic can be observed. Nevertheless, the deviations for $\phi<15^{\circ}$ are obvious and remind us that the SBR itself is an approximation with certain restrictions. This should be kept in mind if additional deviations due to the bistatic approximation are considered. It should be added that the object is fairly small for the SBR and that no diffraction effects are included here.

Concentrating on the effect of the bistatic approximation, it can be seen that the degradation for a bistatic angle $\beta_{\max }=10^{\circ}$ is still very limited. Only a bistatic angle $\beta_{\max } \geq 15^{\circ}$ will cause a considerable impact due to bistatic shadowing.

It should be noted that Fig. 7 is obtained with a high degree of oversampling (see Sect. 4) for visualization purposes. Of course, this is not a sufficient setup for a comparison of the computational efficiency as the improvement that can be observed using the bistatic approximation could also partly be based on averaging the oversampling redundancy. To avoid this, a simulation with the generic object being scaled by a factor of 10 compared to Fig. 6 has been studied. This results in faster oscillations and an increased required sampling rate. For different $\beta_{\max }$ and with varying numbers of rays the deviations of the RCS from the SBR reference, i.e. with a very high number of rays are determined. By comparing the required number of rays for a given level of deviation from the reference the bistatic acceleration can be estimated (see Fig. 8). For $\beta_{\max }=10^{\circ}$ an achievable acceleration factor in the range of $10-20$ can be concluded.

\section{Conclusions}

Based on the principle of monostatic bistatic equivalence, an accelerated ray-based RCS simulation algorithm is proposed and some important implementation details are given. Additional bistatic information, which is collected with very low additional computational cost, can be reused to improve the convergence of the stochastic shooting and bouncing rays algorithm, which means that less rays have to be used for a required accuracy. In this way the monostatic sampling of the object of interest is partly replaced by the usage of bistatic side information. Simulation results are presented for a simple canonical object to demonstrate on the one hand the unavoidable level of degradation that comes along with the usage of a certain amount of bistatic information and how it is related to $\beta_{\max }$. On the other hand the possible speed-up of a complete multi-aspect simulation run is shown by comparing the scattering fields for different number of rays with and without using bistatic information.

From the simulations using the canonical object it can be concluded that, even though this object is shaped quite unfavorable for the application of the bistatic acceleration, the impairment of the monostatic scattering fields is still acceptable for bistatic angles up to 10 or $15^{\circ}$. Therefore a bistatic angle of $10^{\circ}$ can be proposed for the simulation of a passenger car model which will be done in the next future.

It should be noted that for the presented ray-based approach the inaccuracies introduced by the applied assumptions can be expected to be distributed over all observation points, as they are all based on the same ratio of monostatic to bistatic information. Therefore the resulting quality and accuracy of all aspect angles is affected in a similar way. This is in clear contrast to other simulation techniques, where the equivalence principle is used in a post-processing manner to interpolate observation points leading to a reduced accuracy of the interpolated points compared to the directly simulated ones.

Finally, the presented acceleration technique has an advantageous scaling property as it is expected to be most efficient for very high frequencies, where, at the same time, it is needed the most to reduce computational complexity. 


\section{References}

Andersh, D., Moore, J., Bhalla, R., Hughes, J., et al.: Xpatch 4: the next generation in high frequency electromagnetic modeling and simulation software, in: Proc. IEEE International Radar Conference 2000, 844-849, 2000.

Balanis, C. A.: Advanced Engineering Electromagnetics, New York, Wiley, 1989.

Fujimoto, A., Tanaka, T., and Iwata, K.: ARTS: Accelerated Ray Tracing System, IEEE Comput. Graph. Appl., 6(4), April 1986.

Galloway, P. and Welsh, T.: Extensions to the Shooting Bouncing Ray algorithm for scattering from diffuse and grating structures, in: Proc. International Radar Symposium, IRS, Berlin, September 2005.

Kell, R. E.: On the Derivation of Bistatic RCS from Monostatic Measurements, in: Proc. IEEE, vol. 53, August 1965.

Ling, H., Chou, R. C., and Lee, S. W.: Shooting and Bouncing Rays: Calculating the RCS of an Arbitrarily Shaped Cavity, IEEE Trans. Antennas Propag., 37(2), 194-205, 1989.

Mensa, D. L.: High Resolution Radar Cross Section Imaging, Artech House, Boston, 1991.

Schuh, J., Woo, A. C., and Simon, M. P.: The Monostatic/Bistatic Approximation, IEEE Antenn. Propag. M., 36(4), 76-78, 1994.

Siegel, K. M., Crispin, J. W., Newman, J. R., et al.: Methods of Radar Cross-Section Analysis, Academic Press, 1968.

Weinmann, F.: Ray Tracing with PO/PTD for RCS Modeling of Large Complex Objects, IEEE Trans. Antennas Propag., 54(6), 1797-1806, June 2006. 